\title{
Neuronavigation and 3D fluoroscopy-guided lag screw reduction and osteosynthesis for traumatic spondylolistheses of the axis: a path worth exploring?
}

\author{
Jan-Philip Zeden, MD, ${ }^{1}$ Jan-Uwe Müller, MD, ${ }^{1}$ Ehab Ahmed Mohamed El Refaee, MD, ${ }^{1,2}$ \\ Henry W. S. Schroeder, MD, PhD, ${ }^{1}$ and Dirk T. Pillich, MD' \\ 'Department of Neurosurgery, University Medicine Greifswald, Germany; and 2Department of Neurosurgery, Cairo University, \\ Cairo, Egypt
}

\begin{abstract}
OBJECTIVE In traumatic spondylolistheses of the axis, there is a marked heterogeneity of the observed injury patterns, with a wide range of the severity - from stable fractures, which can be treated conservatively with very good success, to highly unstable fractures, which should be treated surgically. A number of classification systems have been devised to assess the instability of the injuries and to derive a corresponding therapy recommendation. In particular, the results and recommendations regarding medium-severity cases are still inconclusive. Minimally invasive percutaneous procedures performed using modern techniques such as 3D fluoroscopy and neuronavigation have the potential for improvements in the therapeutic outcome and procedural morbidity against open surgical procedures and conservative therapy.
\end{abstract}

METHODS A minimally invasive method using 3D fluoroscopy and neuronavigation for percutaneous lag screw osteosynthesis of the pars interarticularis was performed in 12 patients with a Levine-Edwards Type II fracture. Ten patients had an isolated hangman's fracture and 2 patients had an additional odontoid fracture of the axis (Type II according to the Anderson and D'Alonzo classification system). Complications, operating parameters, screw positions, and bony fusion were evaluated for the description and evaluation of the technique.

RESULTS In 6 men and 6 women, percutaneous lag screw osteosynthesis was performed successfully. Correct placement could be verified postoperatively for all inserted screws. In the case series, nonunion was not observed. In all patients with a complete follow-up, a bony fusion, an intact vertebral alignment, and no deformity could be detected on CT scans obtained after 3 months.

CONCLUSIONS The percutaneous pars interarticularis lag screw osteosynthesis is a minimally invasive and mobilitypreserving surgical technique. Its advantages over alternative methods are its minimal invasiveness, a shortened treatment time, and high fusion rates. The benefits are offset by the risk of injury to the vertebral arteries. The lag screw osteosynthesis is only possible with Levine-Edwards Type II fractures, because the intervertebral joints to C-3 are functionally preserved. A further development and evaluation of the operative technique as well as comparison with conservative and alternative surgical treatment options are deemed necessary.

https://thejns.org/doi/abs/10.3171/2017.5.FOCUS17201

KEY WORDS hangman's fracture; Judet osteosynthesis; lag screw osteosynthesis; minimally invasive surgery; traumatic spondylolisthesis of the axis

$\mathrm{T}$ RAUMATIC spondylolisthesis of the axis, or hangman's fracture, is understood as any fracture in which the break passes through the ring of the axis. Axial compression and hyperextension traumas are regarded as the injury mechanism. The most frequent causes are highspeed traumas with a head impact in the context of traffic accidents, falls, or sports accidents.
The fractures show a great variability and can run through the isthmus itself, through the facet joints, or through the corpus of the axis. An asymmetrical fracture configuration is frequently found, which means that the fracture is often hidden in conventional lateral radiographs. On the basis of the analysis of lateral radiographs, classifications of the fractures have been developed. The 

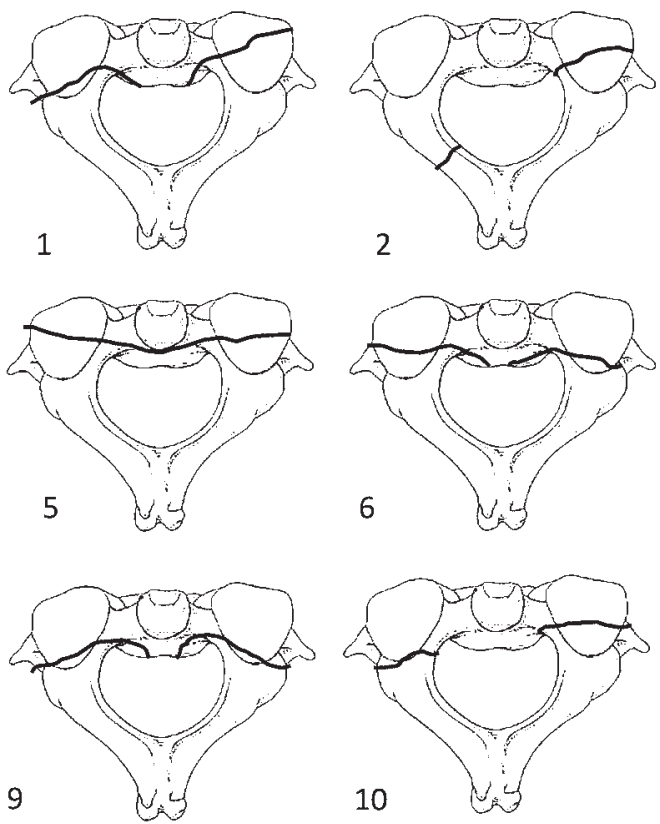

3
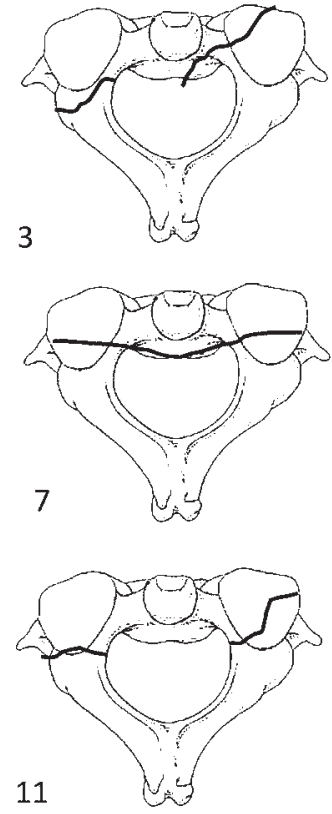
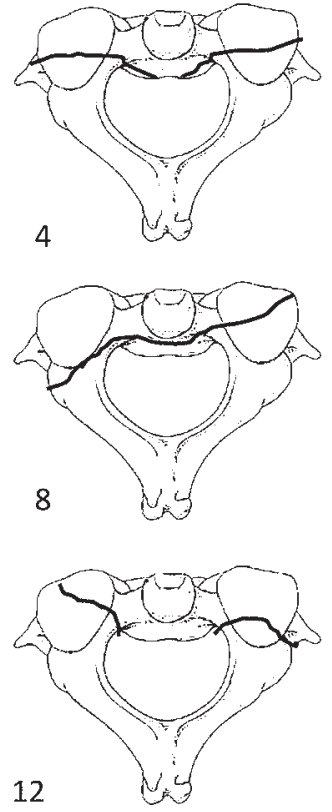

FIG. 1. Drawings showing the fracture sites in the 12 patients in this study.

classifications rely on measurements of the angulation, the translation, the displacement, and the diastasis of the fragments to estimate the severity of the injury and its instability, respectively, to facilitate differentiated therapeutic recommendations.

The most commonly used classification system was developed by Levine and Edwards ${ }^{14}$ and represents an extension of the classification of Effendi et al. ${ }^{5}$ Type I fractures are defined as those with a $<3$-mm displacement without angulation of the fragments. For Type II fractures, the displacement is $>3 \mathrm{~mm}$ and the angulation is $<10^{\circ}$, with a probable rupture of the disc. Type IIa injuries are characterized by a minimal displacement but a high degree of angulation $\left(>10^{\circ}\right)$, indicating a high likelihood of a rupture of the anterior longitudinal ligament. Type III fractures correspond to dislocated Type II fractures, with dislocation or rupture of one or both facet joints, and are therefore associated with the highest instability.

The surgical decision depends on the type and extent of the discoligamentous injury between the axis and C-3. To assess the severity of the injury, knowledge of the injury mechanism as well as additional diagnostics such as MRI or functional radiography of the cervical spine can be helpful. However, no diagnostic tool is sufficiently sensitive to reliably detect discoligamentous injury. $8,10,13,16$

If an instability is diagnosed or probable according to the applied classification, surgical treatment is to be considered and is accepted for cases in which external immobilization is not indicated or has not been successful. ${ }^{18}$ The possible surgical procedures are manifold, and both anterior and posterior stabilizations are established. The anterior interbody fusion of $\mathrm{C} 2 / 3$ has been established as the recommended treatment for the Type IIa lesion (LevineEdwards [L-E] classification). ${ }^{22,23}$ Posterior procedures (among them, most relevant are the $\mathrm{C} 2 / 3$ fusions) are recommended for highly unstable injuries with involvement of the facet joints (L-E Type III). ${ }^{20}$
The lag screw osteosynthesis through the pars interarticularis was first described by Judet et al..$^{12}$ It represents a direct repair of the fracture and thereby restores physiological conditions without segmental fusion. For the conventional (open) approach, the advantages of this physiological repair are canceled out by significant approach-related morbidity. ${ }^{9}$ Therefore, interest in transforming this technique into a minimally invasive procedure arose. The operation is technically difficult due to the individual patient anatomy, the immediate proximity of neurovascular structures, and the unstable fracture conditions. Therefore, high-precision guidance is required for an adaptation of a percutaneous surgical procedure.

Recently, case reports and small case series with minimally invasive approaches have been published. ${ }^{10,11} \mathrm{We}$ present a series of 12 patients in whom a primary operative therapy was indicated or a conservative therapy had failed. All patients were treated with a minimally invasive percutaneous lag screw osteosynthesis through the pars interarticularis under 3D fluoroscopy and neuronavigation by using a precision-optimized technique. The study aimed to evaluate this procedure with regard to risks, technical feasibility, and fusion success.

\section{Methods}

\section{Patient Population}

Percutaneous lag screw osteosynthesis for traumatic spondylolistheses of the axis was performed in 12 patients (6 men, 6 women). All of the injuries were diagnosed on CT scans of the cervical spine. The imaging was further evaluated for CT signs of discoligamentous injury, and the classification system developed by Levine and Edwards was applied. The fractures are illustrated in Fig. 1.

Two patients with a Type II fracture were primarily treated with rigid orthosis and halo vest, respectively. When a pseudarthrosis was diagnosed, surgery was indi- 
TABLE 1. Summary of characteristics and clinical data in 12 patients with traumatic spondylolistheses of the axis

\begin{tabular}{|c|c|c|c|c|c|c|}
\hline Case No. & Etiology & Age (yrs) & Sex & Other Lesions & L-E Type & Indication for Surgery \\
\hline 1 & Fall & 82 & M & Odontoid fracture Type II & II & Surgery indicated \\
\hline 2 & Traffic accident & 53 & M & Head injury & II & Halo traction declined \\
\hline 3 & Stair fall & 82 & M & Head injury & II & Halo traction declined \\
\hline 4 & Stair fall & 54 & $\mathrm{~F}$ & Odontoid fracture Type II & II & Surgery indicated \\
\hline 5 & Traffic accident & 42 & $\mathrm{~F}$ & Unstable fracture T-3 \& T-4 & II & Halo traction contraindicated \\
\hline 6 & Traffic accident & 59 & $\mathrm{~F}$ & Sternum fracture, thorax trauma & II & Halo traction contraindicated \\
\hline 7 & Racing accident & 34 & M & Unstable fracture T-4 \& T-5 & II & Halo traction contraindicated \\
\hline 8 & Epileptic seizure & 49 & $\mathrm{~F}$ & None & II & Pseudarthrosis \\
\hline 9 & Fall & 72 & M & None & II & Malcompliance \\
\hline 10 & Fall & 85 & $\mathrm{~F}$ & None & II & Pseudarthrosis \\
\hline 11 & Fall from height & 79 & M & Sternum fracture & II & Halo traction contraindicated \\
\hline 12 & Fall & 67 & $\mathrm{~F}$ & None & II & Pseudarthrosis \\
\hline
\end{tabular}

cated. In 10 patients, therefore, surgery was the primary management option. The main indications for surgical intervention were significant secondary injuries, necessity of ventilation and intensive care, compliance of the patient, and the patient's preferences.

The causes of injury were vehicle accidents in 4 patients, falling injury in 7 patients, and epileptic seizure in 1 patient. Other associated lesions included odontoid fractures (Type II according to the Anderson and D'Alonzo classification system) (2 patients), head injuries (2 patients), sternum fractures ( 2 patients), and unstable thoracic vertebral fractures (2 patients). A summary of patient data is given in Table 1. All patients were neurologically intact (American Spinal Injury Association Grade E).

\section{Radiological Evaluation}

The fracture evaluation and the application of the L-E classification were based on axial and sagittal CT imaging of the cervical spine. The fracture deformity was described with respect to the angulation C-2 versus C-3, the extent of the displacement, and the translation. The displacement was measured as the maximum distance between the fracture segments. The anterior translation was measured as the distance between a line drawn parallel to the posterior wall of the corpus of $\mathrm{C}-3$ and another line on the posterior wall of the corpus of $\mathrm{C}-2$ on the plane of the disc space. Angulation was measured by the angle derived from the lines drawn along the posterior wall of C-2 and C-3. In addition, imaging was assessed for signs of discoligamentous injuries: CT signs of discoligamentous injury were prevertebral hematoma/edema or tear-drop fracture as indicators for a discontinuity of the anterior longitudinal ligament. A fracture course through the body of C-2 was regarded as a sign of a rupture of the disc and of a decoupling of the anterior and posterior longitudinal ligament; this was present in 5 cases. The former (i.e., prevertebral hematoma/edema or tear-drop fracture) was not observed in the patients in this series. The fracture courses are displayed in Fig. 1.

In all cases, the CT images were loaded into planning software for neuronavigation (Brainlab) to check for the possibility of safe screw placement through the fracture site. Follow-up imaging to evaluate the bony fusion was performed as $\mathrm{CT}$ of the cervical spine. To assess the bony fusion, the imaging was evaluated for new bone formation bridging the fracture gap compared with the prior imaging studies, and the screw seats were assessed for signs of loosening.

\section{Operative Technique}

All patients were intubated by videolaryngoscopy. A carbon Mayfield clamp was affixed and the patient was carefully placed in a Concorde prone position on a carbon table, with a rigid orthosis still applied. The fracture was reduced under lateral fluoroscopy and the head and cervical spine were optimally positioned. After the start of the operation, the reference array for the navigation system (Brainlab, VectorVision) was mounted to the spinous process of the axis (Fig. 2B) through a small midline incision. This was followed by an Iso-C 3D scan (Arcadis, Siemens Medical Solutions) and transfer of the images to the navigation system.

On the basis of the imaging, the trajectories of the lag screws were planned. With a navigated instrument and virtual offset, the entry points were defined and stab incisions were set (Fig. 2A). Afterward, a navigated cannulated instrument was carefully moved to the chosen entry point under navigation guidance and fluoroscopic control (Fig. $2 \mathrm{C})$. After precise positioning and angulation under the guidance of the navigation system, a K-wire was drilled through the fracture site into the corpus of the axis via the cannulated instrument. On the other side the same procedure was followed. A repeated Iso-C 3D scan was performed to verify the correct positioning of the wires. After verification, a pilot bore hole and, if necessary, a threaded cut was performed under fluoroscopic control, depending on the requirements for the screw system. The screw systems used were DensAccess cannulated lag screws (DePuy Synthes), TwinFix cannulated compression screws (Stryker), and Griffin cannulated compression screws (Signus Medizintechnik) (Table 2).

The screws were introduced over the inserted wire and advanced and fastened reciprocally to achieve uniform fracture compression and to avoid deviations of the fragments and screws. Postoperatively a CT scan of the cervi- 

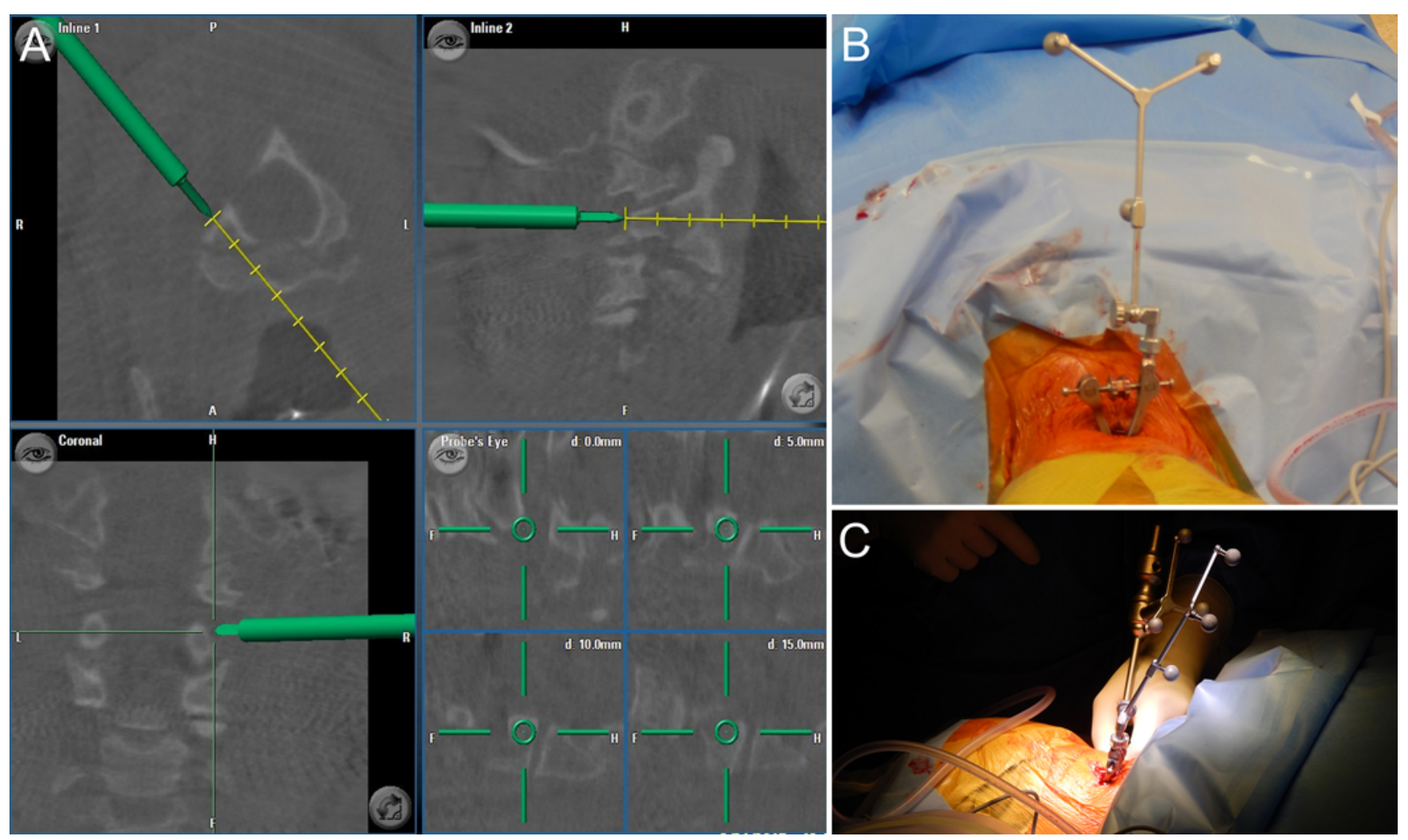

FIG. 2. A: Screenshot of the navigation system showing the setting of the entry point and the angulation of the K-wire. B: Intraoperative photograph of a patient in the prone position, fixed in a carbon Mayfield head clamp. Over a midline incision, the reference array is mounted on the spinous process. C: With virtual offset, the stab incision is placed with a navigated instrument. The entry points are placed with a cannulated navigated instrument, and K-wires are advanced through the fracture site under navigation guidance and fluoroscopic control.

cal spine was performed. Patients wore a rigid orthosis for 6-8 weeks postoperatively.

\section{Results}

In all 12 patients, a satisfactory outcome was achieved. The perioperative radiological data in addition to the clinical condition were evaluated. The average operating time for patients in whom only the bilateral lag screw osteosynthesis was performed was 97 minutes (ranging from 77 to 130 minutes).

The indications for a surgical procedure were, in 2 cases, a higher degree of instability due to an additional odontoid fracture (Anderson and D'Alonzo Type II). In 4 patients, halo traction was not indicated due to concomi-

TABLE 2. Summary of surgical and follow-up data in 12 patients with traumatic spondylolistheses of the axis

\begin{tabular}{|c|c|c|c|c|c|}
\hline $\begin{array}{l}\text { Case } \\
\text { No. }\end{array}$ & Procedure & $\begin{array}{l}\text { Screw } \\
\text { Type }\end{array}$ & $\begin{array}{l}\text { Op Time } \\
\text { (mins) }\end{array}$ & $\begin{array}{l}\text { Follow-Up } \\
\text { (days) }\end{array}$ & $\begin{array}{l}\text { Fusion } \\
\text { Status }\end{array}$ \\
\hline 1 & Bilat lag screw osteosynthesis \& anterior odontoid lag screw osteosynthesis & TwinFix & 225 & 80 & Bony fusion \\
\hline 2 & Lt unilat lag screw osteosynthesis & TwinFix & 77 & 94 & Bony fusion \\
\hline 3 & Bilat lag screw osteosynthesis & DensAccess & 130 & 94 & Bony fusion \\
\hline 4 & Bilat lag screw osteosynthesis \& anterior odontoid lag screw osteosynthesis & DensAccess & 169 & 71 & Bony fusion \\
\hline 5 & Bilat lag screw osteosynthesis \& posterior bisegmental screw rod fracture stabilization & DensAccess & 198 & 90 & Bony fusion \\
\hline 6 & Bilat lag screw osteosynthesis & Griffin & 100 & 226 & Bony fusion \\
\hline 7 & Bilat lag screw osteosynthesis \& posterior bisegmental screw rod fracture stabilization & DensAccess & 297 & 102 & Bony fusion \\
\hline 8 & Bilat lag screw osteosynthesis & TwinFix & 95 & 96 & Bony fusion \\
\hline 9 & Bilat lag screw osteosynthesis & DensAccess & 108 & 81 & Bony fusion \\
\hline 10 & Bilat lag screw osteosynthesis & Griffin & 92 & 135 & Bony fusion \\
\hline 11 & Bilat lag screw osteosynthesis & DensAccess & 77 & 132 & Bony fusion \\
\hline 12 & Bilat lag screw osteosynthesis & DensAccess & 79 & 75 & Bony fusion \\
\hline
\end{tabular}


tant injuries of the thorax. Two patients underwent operation because they rejected halo immobilization and preferred the surgical intervention. One patient with existing delirium was surgically treated due to lack of compliance with conservative therapy. In 2 other patients, conservative therapy with rigid orthosis or halo vest had failed. In 1 patient no medical consultation had taken place after the trauma, and the diagnosis by imaging was delayed. Because of the long interval between the trauma and medical attention, surgical therapy was indicated (Table 2). No intraoperative complications were encountered. The postoperative $\mathrm{CT}$ imaging confirmed the correct position of the traction screws in all cases without affecting the vertebral canal. The mean fracture reduction was $2 \mathrm{~mm}$ (range $1-5$ $\mathrm{mm}$ ) compared with preoperative imaging. Surgical revisions were not required. The loss of blood during lag screw osteosynthesis was negligible. Wound healing problems were not observed.

Bony fusion was considered to be present when CT imaging showed evidence of healing across the fracture site and absent signs of material failure. The mean followup period was 106 days, ranging from 71 to 226 days, at which time bony fusion was confirmed in all cases by a CT scan (Fig. 3). All patients experienced a smooth follow-up with no new neurological deficit after the operation and improvement of the neck pain.

\section{Discussion}

The majority of patients with traumatic spondylolistheses of the axis can be successfully treated conservatively with a cervical orthosis or with halo traction after closed reduction. The reported fusion rates after conservative therapy in the large case series are high. However, in these series, injuries with a higher degree of instability are more likely to lead to a failure of conservative therapy, with the need for surgical treatment. In the series by Greene et al., due to insufficient immobilization in the halo vest, 7 of 28 patients with Effendi Grade II and III injuries had to be treated surgically. ${ }^{9}$ Francis et al. reported a failure of fracture consolidation of $28 \%$ and $33 \%$ in patients assigned into medium and high grades of instability, respectively, according to their own classification. ${ }^{7}$ In a meta-analysis, L-E Type II injuries could be treated conservatively in $60 \%$ of cases. ${ }^{15}$ On the other hand, the reported fusion rates of surgically treated cases are almost $100 \%$. In addition to the lower fusion rates of the injuries that are classified as unstable, there are further disadvantages of conservative therapy in its longer duration, and the discomfort or the not-infrequent complications of halo therapy. ${ }^{14}$ In the spectrum of injuries, conservative therapy of a stable L-E Type I injury is the first choice. In contrast, surgical therapy is established as the recommended treatment for highly unstable fractures such as L-E Types IIa and III. For L-E Type II fractures, the existing literature is inconclusive.

Several previous studies have advocated that the primary therapy for all hangman's fractures should be conservative. ${ }^{15}$ On the contrary, Shin et al. described posterior reduction and screw fixation as a primary treatment strategy in all patients with hangman's fracture of all grades. ${ }^{19}$ It was previously reported that stable fractures (L-E Type
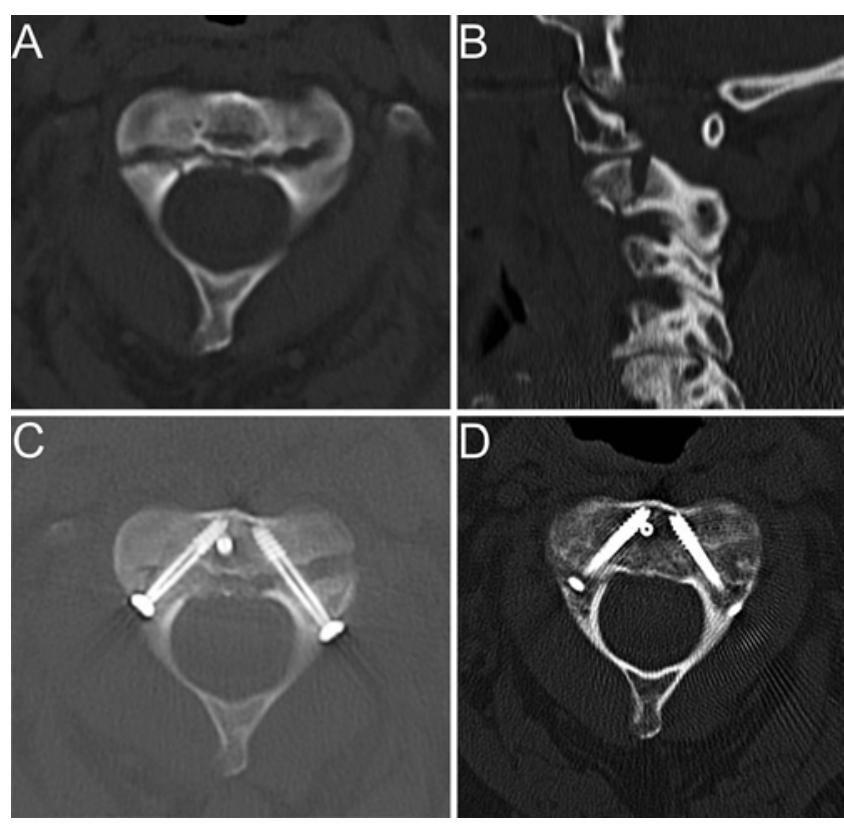

FIG. 3. Case 4. A: Preoperative axial CT scan. B: Preoperative sagittal CT scan. C: Postoperative axial CT scan. D: Axial CT scan obtained after 3 months, demonstrating consolidated fracture.

I) and certain adequately reduced L-E Type II fractures are generally treated with brace therapy involving a rigid cervical collar or halo immobilization. ${ }^{14}$ For unstable fractures, including some L-E Type II and most Type IIa and III fractures, if severe dislocation is present or when external brace immobilization fails, surgical management is generally indicated..$^{5,21}$ The question of the inherent stability of Type II fractures is not solved by the L-E classification, just as it is not solved with other classification systems. Among these fracture types are relatively stable or reduced injuries with lower displacement, translation, and angulation, and consequently a low probability of a ruptured disc and ligamentous injury. These cases can be treated with a rigid orthosis. For injuries with a high probability of a ruptured disc and/or a ruptured posterior longitudinal ligament, external traction and immobilization with a halo vest or surgical therapy is indicated. This differentiated approach to this type of injury is also advocated by others.,11,17

The halo vest leads to an improved immobilization of the upper cervical spine and enables fracture reduction. Halo therapy and surgical stabilization are therefore regarded as complementary for unstable L-E Type II injuries. ${ }^{1,17}$

Alternative operative procedures are the posterior stabilization of $\mathrm{C} 2 / 3$, and in particular the anterior intercorporal fusion of $\mathrm{C} 2 / 3$ with plating. The open posterior procedures have several disadvantages compared with direct lag screw osteosynthesis. The surgery-related morbidity of the open posterior approaches, with increased postoperative pain, the higher risk of infection, the increased blood loss, and the longer recovery phase after the procedure is one of those disadvantages. The anterior intercorporal fusion is an operating technique that is common to spine surgeons, although the necessary plating in this segment 
is frequently not problem free in the implementation, and therefore entails an increased complication risk. ${ }^{23}$

Posterior lag screw osteosynthesis aims to combine the advantages of surgical therapy with regard to the high fusion rates and the advantages of conservative therapy with regard to functional preservation and low therapy-related morbidity. However, it is feared that the possible benefits are offset by the risk of injury to the vertebral arteries. The placement of direct C-2 pars or pedicle screws is technically challenging because of potentially variable anatomy, especially in patients with an already injured spine. The screw would pass through the narrowest part of the vertebra, medially bounded by the spinal cord and laterally by the vertebral artery. Open procedures for the management of hangman's fractures have been previously described, ranging from transoral discectomy and anterior plate fixation to open, posterior, C-2 pedicle screw fixation. ${ }^{6}$ In a previous small series, CT-guided neuronavigation was implemented to perform the transpedicular C-2 fixation percutaneously. ${ }^{3}$ The intraoperative navigation diminished the possibility of placement inaccuracies, so that injury to the vertebral artery and of the neural tissue could be prevented.

The positioning of the dynamic reference frame is a decisive factor for the accuracy of the navigation. Some authors prefer fixation to the head clamp. ${ }^{3}$ A rationale for this is the pathological mobility of the spinous process of the axis, possibly leading to a deviation of navigation during the instrumentation. In this case series, fixation of the dynamic reference frame on the spinous process of C-2 was preferred. The accuracy of the navigation is of utmost importance for the placement of the guide wires. Under loading on the entry point at the isthmus, which inevitably occurs, a rotational movement of the axis occurs even at low forces, which in turn requires a significant correction of the angulation. A fixation of the reference (star) on the headholder does not show this movement and leads to inaccuracy of the system. In this context, it is important to consider the extent to which instability of the fracture leads to a different deviation of the anterior fragment (the corpus) from the posterior fragment when a force is applied during the instrumentation. Therefore the close combination of navigation with fluoroscopy remains indispensable.

The fusion rates of lag screw osteosyntheses are comparable to other operative procedures, despite limited evidence due to the low number of cases in the reported small case series..$^{2-4,6,21}$ Verheggen et al. demonstrated preservation of the physiological mobility of the upper cervical spine after this procedure. ${ }^{21}$

In the cases in this series, we opted for rigid orthosis postoperatively. Alternatively, flexion-extension radiographs could be obtained 2 weeks postoperatively to rule out remaining instability due to an occult rupture of the anterior longitudinal ligament, to further reduce the immobilization time.

The advantage of the case series presented here is the validation of the bony fusion by CT imaging of the cervical spine. Here, bony consolidations or their absence and the strength of the osteosynthesis material can be assessed more sensitively and more specifically than in lateral radiographic images with and without functional imaging. According to our results, percutaneous navigation-guided pedicle screw placement in the axis is considered to be a safe procedure that leads to high fusion rates. To our knowledge, this clinical series represents the largest to date that analyzes the outcome after navigation-guided transpedicular osteosynthesis in patients with hangman's fractures.

\section{Conclusions}

The percutaneous pars interarticularis lag screw osteosynthesis procedure is a minimally invasive and mobility-preserving surgical technique. Its advantages over alternative methods are its minimal invasiveness, a shortened treatment time, and high fusion rates. The navigation guidance serves to minimize the risk of neural or vertebral artery injury. The lag screw osteosynthesis technique is possible with L-E Type II fractures, because the intervertebral joints to C-3 are functionally preserved. Further development and evaluation of the operative technique as well as comparison with conservative and alternative surgical treatment options are encouraged.

\section{References}

1. Al-Mahfoudh R, Beagrie C, Woolley E, Zakaria R, Radon M, Clark S, et al: Management of typical and atypical hangman's fractures. Global Spine J 6:248-256, 2016

2. Boullosa JLR, Colli BO, Carlotti CG Jr, Tanaka K, dos Santos MB: Surgical management of axis' traumatic spondylolisthesis (Hangman's fracture). Arq Neuropsiquiatr 62 (3B):821-826, 2004

3. Buchholz AL, Morgan SL, Robinson LC, Frankel BM: Minimally invasive percutaneous screw fixation of traumatic spondylolisthesis of the axis. J Neurosurg Spine 22:459465, 2015

4. Dalbayrak S, Yilmaz M, Firidin M, Naderi S: Traumatic spondylolisthesis of the axis treated with direct $\mathrm{C} 2$ pars screw. Turk Neurosurg 19:163-167, 2009

5. Effendi B, Roy D, Cornish B, Dussault RG, Laurin CA: Fractures of the ring of the axis. A classification based on the analysis of 131 cases. J Bone Joint Surg Br 63-B:319-327, 1981

6. ElMiligui Y, Koptan W, Emran I: Transpedicular screw fixation for type II Hangman's fracture: a motion preserving procedure. Eur Spine J 19:1299-1305, 2010

7. Francis WR, Fielding JW, Hawkins RJ, Pepin J, Hensinger R: Traumatic spondylolisthesis of the axis. J Bone Joint Surg Br 63-B:313-318, 1981

8. Goradia D, Linnau KF, Cohen WA, Mirza S, Hallam DK, Blackmore CC: Correlation of MR imaging findings with intraoperative findings after cervical spine trauma. AJNR Am J Neuroradiol 28:209-215, 2007

9. Greene KA, Dickman CA, Marciano FF, Drabier JB, Hadley MN, Sonntag VK: Acute axis fractures. Analysis of management and outcome in 340 consecutive cases. Spine (Phila Pa 1976) 22:1843-1852, 1997

10. Insko EK, Gracias VH, Gupta R, Goettler CE, Gaieski DF, Dalinka MK: Utility of flexion and extension radiographs of the cervical spine in the acute evaluation of blunt trauma. J Trauma 53:426-429, 2002

11. Josten C: Die traumatische Spondylolisthese des Axis. Orthopade 28:394-400, 1999

12. Judet J, Roy-Camille R, Rose B, Feghali G, Bedoiseau M, Saillant G: [Tetraplegia caused by fracture-dislocations of the cervical spine treated by emergency reduction and osteosynthesis.] J Chir (Paris) 101:249-258, 1971 (Fr)

13. Khan SN, Erickson G, Sena MJ, Gupta MC: Use of flexion and extension radiographs of the cervical spine to rule out acute instability in patients with negative computed tomography scans. J Orthop Trauma 25:51-56, 2011 
14. Levine AM, Edwards CC: The management of traumatic spondylolisthesis of the axis. J Bone Joint Surg Am 67:217226, 1985

15. Li XF, Dai LY, Lu H, Chen XD: A systematic review of the management of hangman's fractures. Eur Spine J 15:257269, 2006

16. Malham GM, Ackland HM, Varma DK, Williamson OD: Traumatic cervical discoligamentous injuries: correlation of magnetic resonance imaging and operative findings. Spine (Phila Pa 1976) 34:2754-2759, 2009

17. Müller EJ, Wick M, Muhr G: Traumatic spondylolisthesis of the axis: treatment rationale based on the stability of the different fracture types. Eur Spine J 9:123-128, 2000

18. Ryken TC, Hadley MN, Aarabi B, Dhall SS, Gelb DE, Hurlbert RJ, et al: Management of isolated fractures of the axis in adults. Neurosurgery 72 (Suppl 2):132-150, 2013

19. Shin JJ, Kim SH, Cho YE, Cheshier SH, Park J: Primary surgical management by reduction and fixation of unstable hangman's fractures with discoligamentous instability or combined fractures. Spine (Phila Pa 1976) 19:569-575, 2013

20. Tian W, Weng C, Liu B, Li Q, Hu L, Li ZY, et al: Posterior fixation and fusion of unstable Hangman's fracture by using intraoperative three-dimensional fluoroscopy-based navigation. Eur Spine J 21:863-871, 2012

21. Verheggen R, Verheggen R, Jansen J, Jansen J: Hangman's fracture: arguments in favor of surgical therapy for type II and III according to Edwards and Levine. Surg Neurol 49:253-262, 1998
22. Xu H, Zhao J, Yuan J, Wang C: Anterior discectomy and fusion with internal fixation for unstable hangman's fracture. Int Orthop 34:85-88, 2010

23. Ying Z, Wen Y, Xinwei W, Yong T, Hongyu L, Zhu H, et al: Anterior cervical discectomy and fusion for unstable traumatic spondylolisthesis of the axis. Spine (Phila Pa 1976) 33:255-258, 2008

\section{Disclosures}

The authors report no conflict of interest concerning the materials or methods used in this study or the findings specified in this paper.

\section{Author Contributions}

Conception and design: Zeden, Müller. Acquisition of data: Zeden. Analysis and interpretation of data: Zeden. Drafting the article: Zeden, El Refaee. Critically revising the article: Müller, El Refaee, Schroeder, Pillich. Reviewed submitted version of manuscript: Schroeder, Pillich. Administrative/technical/material support: Müller. Study supervision: Schroeder, Pillich.

\section{Correspondence}

Jan-Philip Zeden, University Medicine Greifswald, Sauerbruchstrasse, Greifswald 17489, Germany. email: jpzeden@ uni-greifswald.de. 